\title{
Scalable Heating-Up Synthesis of Monodisperse Cu2ZnSnS4
}

\section{Nanocrystals}

\author{
Alexey Shavel, ${ }^{\dagger}, \hbar, *$ Maria Ibáñez, ${ }^{\dagger}$ Zhishan Luo,${ }^{\dagger}$ Jonathan De Roo, ${ }^{\ddagger}$ Alex Carrete, ${ }^{\dagger}$ Mirjana \\ Dimitrievska, ${ }^{\dagger}$ Aziz Genç, ${ }^{\mathbb{I}}$ Michaela Meyns,${ }^{\dagger}$ Alejandro Perez-Rodriguez, ${ }^{\dagger, \dagger}$ Maksym V. Kovalenko, ${ }^{\perp, \|}$ \\ Jordi. Arbiol, ${ }^{\mathrm{I}, \S}$ and Andreu Cabot, ${ }^{\dagger}, \S$ \\ ${ }^{\dagger}$ Catalonia Energy Research Institute - IREC, Sant Adrià del Besòs, Barcelona, 08930, Spain. \\ "I Institut Català de Nanociència i Nanotecnologia, ICN2, Campus de la UAB, 08193 Bellaterra, Spain. \\ *Departament d'Electrònica, Universitat de Barcelona, 08028 Barcelona, Spain. \\ §Institució Catalana de Recerca i Estudis Avançats - ICREA, 08010 Barcelona, Spain. \\ ${ }^{¥}$ Ghent University, Department of Inorganic and Physical Chemistry, Krijgslaan 281 S3, 9000 Gent, Belgium. \\ ${ }^{\perp}$ Institute of Inorganic Chemistry, Department of Chemistry and Applied Biosciences, ETH Zürich, CH-8093, Switzerland. \\ "Empa-Swiss Federal Laboratories for Materials Science and Technology, Dübendorf, CH-8600, Switzerland.
}

\begin{abstract}
Monodisperse $\mathrm{Cu}_{2} \mathrm{ZnSnS}_{4}$ (CZTS) nanocrystals were prepared by a facile, high yield, scalable and high concentration heating-up procedure. An efficient mixing and heat transfer in the reaction mixture using intensive argon bubbling and a careful control of the heating ramp stability were key parameters to minimize the nanocrystal size distribution. Optimized synthesis conditions allowed the production of several grams of highly monodisperse CZTS nanocrystals per batch, with a $5 \mathrm{wt} \%$ concentration and a yield above $90 \%$.
\end{abstract}

\section{INTRODUCTION}

Ternary and quaternary semiconductors have recently attracted a lot of attention in the field of renewable energy conversion, and particularly in photovoltaics, photocatalysis and thermoelectrics. ${ }^{1-11}$ Among them, $\mathrm{Cu}_{2} \mathrm{ZnSnS}_{4}$ (CZTS), comprising abundant and non-toxic elements, has a high absorption coefficient in the visible region $\left(>10^{4} \mathrm{~cm}^{-1}\right)$ and a suitable band gap (1.45-1.50 eV) for solar energy conversion. These properties make it an excellent absorber material for the next generation of solar cells ${ }^{1}$ and photocatalysts. ${ }^{6-9}$

The availability of these quaternary semiconductors in the form of nanocrystal-based inks offers the opportunity to lowcost solution processing by common techniques such as spraycoating or inkjet printing. Inexpensive and scalable bottom-up approaches are increasingly needed for transferring the compositional versatility and homogeneity of individual nanocrystals to the device-level macroscopic materials, either as large area thin films or bulk-like nanogranular materials. ${ }^{12-}$

However, the preparation of multinary nanocrystals presents a much higher level of difficulty than the synthesis of elemental and binary nanocrystals. In particular, formation of quaternary chalcogenides requires narrow parametric spaces and competes with simultaneous formation of binary and ternary phases. To this end, diversity of phases and compositions in a mixture of several elements also offers important advantages. For instance, $\mathrm{Cu}$-poor and $\mathrm{Zn}$-rich CZTS materials have demonstrated better performances as absorbers in solar cells than nearly stoichiometric materials. On the other hand, Cu-rich CZTS have shown improved photocatalytic properties. ${ }^{6}$ Furthermore, the compositional control allows tuning of the semiconductor charge carrier concentration, which is key to optimize performance in applications such as thermoelectricity. ${ }^{15}$
Clearly, control and analysis of compositional and phase homogeneities are experientially highly challenging and require high spatial resolution chemical analysis tools such as energy-dispersive X-ray spectroscopy (EDX), electron energy loss spectroscopy (EELS) or Raman spectroscopy. On ensemble level, oftentimes shape and size-uniformity of multinary nanocrystals are indicative of their intrinsic chemical homogeneity. ${ }^{16-19}$ In other words, monodisperse nanocrystals are more likely to be also chemically identical.

Since photovoltaic, catalytic and nearly all other applications require large quantities of nanocrystals with a minimal particle-to-particle variation in composition, the aim of this work was to develop a high yield and easily scalable synthesis protocol to produce uniform CZTS nanocrystals at the multigram scale. The 'heating-up' synthesis - when all reagents and solvents are pre-mixed and then simply heated is generally easier to upscale than the injection-based procedures, primarily due to highly homonegenous concentrations and temperatures throughout the process. ${ }^{20}$ When the injection of a reagent is involved, upscaling is limited by difficulties to achieve fast mixing of large volumes of reagents. Few gram-scale synthesis protocols to produce CZTS nanocrystals have been already proposed. Among them, the solvent-free, mechanochemical synthesis approach is a remarkably simple pathway, ${ }^{21}$ albeit with limited control over the composition and morphology of CZTS nanocrystals.

Here we report a $90 \%$ yield synthesis of monodisperse CZTS nanocrystals using a heating-up procedure and high initial concentration of precursors, up to $0.6 \mathrm{mmol}$ per gram of a reaction mixture, that is a factor of 8-10 higher than commonly used in nanocrystal synthesis. We also present a detailed nuclear magnetic resonance (NMR) study of the surface capping ligands and an efficient organic ligand displacement procedure, with final carbon content of just 0.1 $0.2 \mathrm{w} \%$. 


\section{EXPERIMENTAL SECTION}

Chemicals and solvents: Tert-dodecylmercaptan ( $t$-DDT, 98.5\%), 1-dodecanethiol (1-DDT, $\geq 98 \%$ ), tin (IV) chloride pentahydrate $\left(\mathrm{SnCl}_{4} \cdot 5 \mathrm{H}_{2} \mathrm{O}, 98 \%\right)$, zinc oxide $(\mathrm{ZnO}, \geq 99 \%)$, copper (II) chloride dihydrate $\left(\mathrm{CuCl}_{2} \cdot 2 \mathrm{H}_{2} \mathrm{O}, \geq 99 \%\right)$, 1 octadecene (ODE, 90\%), and oleylamine (OLA, 70\%) were purchased from Aldrich. Chloroform, isopropanol, dimethyl sulfoxide (DMSO) and tetrahydrofuran (THF) were of analytical grade and obtained from various sources. OLA was vacuum distilled before its use. The use of 'as received' OLA results in poor nanocrystal size distributions and irregular nanocrystal shapes. Others precursors and solvents were used without further purification. Cole-Parmer 'Digi-Sense R/S' temperature controllers with enabled 'auto-tuning' mode were used for the accurate control of the heating rate during the synthesis.

CZTS nanocrystals: Quasi-spherical CZTS nanocrystals were prepared by the reaction of copper and tin chlorides and zinc oxide with a mixture of tert-dodecylmercaptan and dodecanethiol in the presence of OLA. In a typical synthesis, $5.4 \mathrm{mmol}(920 \mathrm{mg})$ of $\mathrm{CuCl}_{2} \cdot 2 \mathrm{H}_{2} \mathrm{O}, 4.8 \mathrm{mmol}(391 \mathrm{mg})$ of $\mathrm{ZnO}$ and $1.8 \mathrm{mmol}(630 \mathrm{mg})$ of $\mathrm{SnCl}_{4} \cdot 5 \mathrm{H}_{2} \mathrm{O}$ were dissolved in a minimum amount of THF. Afterward, $24 \mathrm{mmol}(6.43 \mathrm{~g})$ of distilled OLA and $20 \mathrm{~g}$ of ODE were added to the reaction mixture. The solution was heated under argon flow (ca. 30 $\mathrm{mL} / \mathrm{min}$ ) to $175{ }^{\circ} \mathrm{C}$ and maintained at this temperature for approximately 1 hour to ensure the removal of traces of low boiling point impurities and water. Three issues to be noted at this point: i) the purification time may be significantly optimized in a large scale production scenario; ii) the main reason to use THF as co-solvent is to facilitate the metal salts dissolution, but it also helped to remove water from the reaction mixture; iii) $\mathrm{ZnO}$ is soluble only with the presence of $\mathrm{SnCl}_{4} \cdot 5 \mathrm{H}_{2} \mathrm{O}$ in a solution.

After purging, the mixture was cooled to $100{ }^{\circ} \mathrm{C}$ and 50 mmol (10.12 g) of tert-dodecylmercaptan and $5 \mathrm{mmol}(1.012$ g) of dodecanethiol were added though a syringe. Then the solution was heated to $250{ }^{\circ} \mathrm{C}$ during 10 minutes and maintained at this temperature for 1 hour. A constant heating rate and a high argon flow during the heating were key be obtain monodisperse nanocrystals. Formation of nanocrystals was noticed by a gradual darkening of the reaction mixture at about $135-145^{\circ} \mathrm{C}$. Final CZTS nanocrystals were thoroughly purified by multiple precipitation and re-dispersion steps using 2-propanol and chloroform. Finally, CZTS nanocrystals were dissolved in THF and the solution was centrifuged at 9000 rpm during 5-15 minutes to precipitate poorly soluble unreacted metal complexes and large (> $100 \mathrm{~nm})$ Zn-rich particles (note the initial non-stoichiometric precursor ratio). The supernatant was recovered and stored for its posterior use. No additional size selective precipitation was performed. The proposed synthetic route allowed to prepare about $670 \mathrm{mg}$ of CZTS nanocrystals in $30 \mathrm{~g}$ of reaction mixture, and with a precursor metal concentration of $0.3 \mathrm{mmol} / \mathrm{g}$. It is worth mentioning that total precursors concentration might be doubled simply by reducing the amount of solvent. However, by doing so the viscosity of the reaction mixture increased significantly, reducing the product quality and rendering the synthetic procedure less convenient. Instead, we propose to proportionally scale up the synthesis a factor 10 by conducting the synthesis in a 1 liter flask. In fact, we regularly performed this synthesis protocol at 5-7 fold scale, producing 5-6 $\mathrm{g}$ of CZTS nanocrystals per batch with essentially the same results. The typical yield of the synthesis was about $90 \%$.
Characterization techniques: The morphological, chemical and structural characterizations of the nanocrystals were carried out by transmission electron microscopy (TEM) and high-resolution TEM (HRTEM). Carbon-coated TEM grids from Ted-Pella were used as substrates. A solution of the CZTS nanocrystals in THF was directly dropped on the grids and dried at the ambient condition. HRTEM images were obtained using a FEI Tecnai F20 field-emission gun microscope operated at $200 \mathrm{keV}$ with an embedded Gatan image filter for EELS analyses. Images were analyzed by means of Quantum Gatan micrograph software. Powder X-ray diffraction (XRD) patterns were obtained with $\mathrm{Cu} \mathrm{K} \mathrm{K}_{\alpha 1}(\lambda=$ $1.5406 \AA$ ) radiation in a reflection geometry on a Bruker D8 operating at $40 \mathrm{kV}$ and $40 \mathrm{~mA}$. Raman scattering measurements were performed in backscattering configuration with a LabRam HR800-UV Horiba-Jobin Yvon spectrometer, using the $532 \mathrm{~nm}$ line from a Nd:YAG solid state laser and the $325.0 \mathrm{~nm}$ line from a HeNe laser as excitation lights. UV-vis optical absorption spectra were recorded on a LAMBDA 950 UV-Vis spectrophotometer from PerkinElmer. Elemental analyses were performed using an elemental organic analyzer Thermo EA 1108, working under $120 \mathrm{ml} / \mathrm{min}$ helium flow, the combustion furnace at $1000{ }^{\circ} \mathrm{C}$, the chromatographic column oven at $60{ }^{\circ} \mathrm{C}$, and $10 \mathrm{ml}$ oxygen loop at $100 \mathrm{kPa}$.

Nuclear Magnetic Resonance (NMR) measurements were recorded on a Bruker Avance III HD Spectrometer operating at a ${ }^{1} \mathrm{H}$ frequency of $500.26 \mathrm{MHz}$ and equipped with a BBFO$\mathrm{Z}$ probe. The sample temperature was set to $298.2 \mathrm{~K}$. One dimensional (1D) ${ }^{1} \mathrm{H}$ and 2D NOESY (Nuclear Overhauser Effect Spectroscopy) spectra were acquired using standard pulse sequences from the Bruker library. For the quantitative $1 \mathrm{D}{ }^{1} \mathrm{H}$ measurements, $64 \mathrm{k}$ data points were sampled with the spectral width set to $20 \mathrm{ppm}$ and a relaxation delay of $30 \mathrm{sec}$. The NOESY mixing time was set to $300 \mathrm{~ms}$ and 4096 data points in the direct dimension for 512 data points in the indirect dimension were sampled, with the spectral width set to $12 \mathrm{ppm}$. Diffusion measurements (2D DOSY) were performed using a double stimulated echo sequence for convection compensation and with monopolar gradient pulses. ${ }^{24}$ Smoothed rectangle gradient pulse shapes were used throughout. The gradient strength was varied quadratically from $2-95 \%$ of the probe's maximum value in 64 increments, with the gradient pulse duration and diffusion delay optimized to ensure a final attenuation of the signal in the final increment of less than $10 \%$ relative to the first increment. For 2D processing, the spectra were zero filled until a 4096 - 2048 real data matrix. Before Fourier transformation, the 2D spectra were multiplied with a squared cosine bell function in both dimensions, the 1D spectra were multiplied with an exponential window function. Concentrations were obtained using the Digital ERETIC method, as provided in the standard software of Bruker. The diffusion coefficients were obtained by fitting the appropriate Stejskal-Tanner equation to the signal intensity decay. ${ }^{25}$

\section{RESULTS AND DISCUSSION}

Representative TEM and HRTEM micrographs of the CZTS nanocrystals prepared following the above detailed heating-up procedure are shown on figures $1 \mathrm{a}$ and $1 \mathrm{~b}$ respectively. CZTS nanocrystals have slightly faceted, nearly isotropic spherical shapes. Importantly, the size dispersion was typically below $10 \%$ (estimated as full-width at half maximum of the size histogram collected from several hundred nanocrystals). The power spectrum, shown in figure $1 \mathrm{~b}$, unambiguously revealed CZTS nanocrystals with a wurtzite phase and lattice 
parameters; $\mathrm{a}=\mathrm{b}=0.3839 \mathrm{~nm}$ and $\mathrm{c}=0.6339 \mathrm{~nm}\left(\mathrm{P}_{3} \mathrm{mc}\right.$ space group). XRD analysis confirmed the wurtzite crystal phase of the CZTS nanocrystals (figure 1c). The presence of alkylthiols in the reaction mixture was found to be crucial to obtain CZTS nanocrystals of this crystal structure. Contrarily, using sulfur as a precursor leads to the formation of nanoparticles with kesterite structure. ${ }^{30}$ Raman spectra of the produced materials are shown in figure 1d. Various excitation wavelengths were used to enhance the signal of the possible secondary and ternary phases. The Raman spectrum obtained
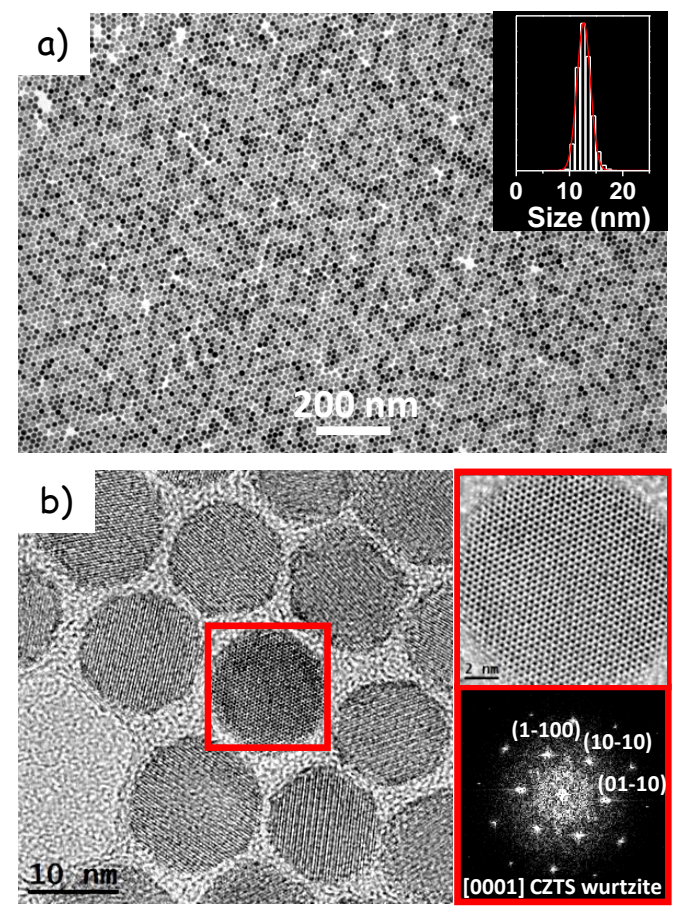

with a $532 \mathrm{~nm}$ excitation shows two broad bands at $333 \mathrm{~cm}^{-1}$ and $288 \mathrm{~cm}^{-1}$, which agree well with the reported spectra of wurtzite CZTS. The presence of $\mathrm{CuS}, \mathrm{SnS}, \mathrm{SnS}_{2}, \mathrm{SnS}_{3}$ or $\mathrm{Cu}_{2} \mathrm{SnS}_{3}$ would have associated bands at $475 \mathrm{~cm}^{-1}, 190-220$ $\mathrm{cm}^{-1}, 315 \mathrm{~cm}^{-1}, 310 \mathrm{~cm}^{-1}$ or $350 \mathrm{~cm}^{-1}$, respectively, which were not observed. ${ }^{19}$ Raman spectra with $325 \mathrm{~nm}$ excitation were also obtained to identify the presence of $\mathrm{ZnS}$, which is characterized by the presence of two peaks at $350 \mathrm{~cm}^{-1}$ and $700 \mathrm{~cm}^{-1}$. None of these peaks was observed, ruling out the presence of this phase in the final material.
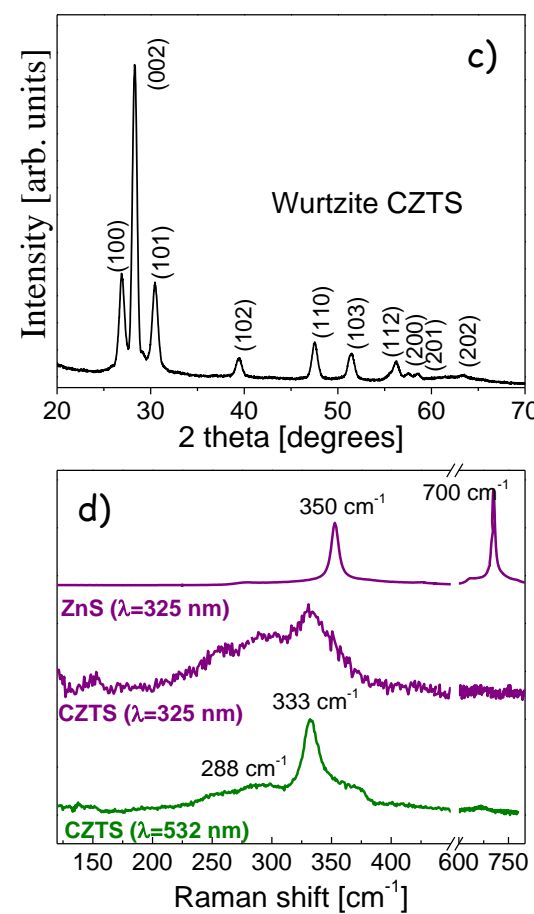

Figure 1. a) Representative TEM micrograph and typical size-histogram of CZTS nanocrystals; b) HRTEM micrograph and corresponding indexed power spectrum on the selected red squared CZTS nanocrystal; c) XRD pattern and d) Raman spectra with $532 \mathrm{~nm}$ and $325 \mathrm{~nm}$ excitation of CZTS nanocrystals. The resonant Raman spectrum of ZnS obtained under $325 \mathrm{~nm}$ excitation is also shown as a reference.

Figure 2 shows the programed temperature profiles and actual read-outs from the thermocouples during the synthesis. The initial formation of nanocrystals was observed by a clear color change in the reaction mixture from yellow to darkbrown at $135-145{ }^{\circ} \mathrm{C}$. At this point, copper sulfide nanocrystals nucleated first due to high chalcophilicity of this metal. These nanocrystals then grew by incorporation of all present metals $(\mathrm{Cu}, \mathrm{Zn}$ and $\mathrm{Sn}$ ions) as the temperature increases. A similar mechanism of the formation of quaternary phases, based on the progressive incorporation of the different cations in an initially formed copper chalcogenide lattice, was observed for several $\mathrm{Cu}$-based quaternary chalcogenide semiconductors such as $\mathrm{Cu}_{2} \mathrm{CdSnSe}_{4},{ }^{26}$ and $\mathrm{CZTS}^{27-29}$ The formation of the CZTS nanocrystals was completed close to the reaching of the peak temperate of the temperature $\operatorname{ramp}\left(250{ }^{\circ} \mathrm{C}\right)$. Finally, the obtained composition and size remained unchanged at after additional anneal at $250{ }^{\circ} \mathrm{C}$ for 30 minutes.
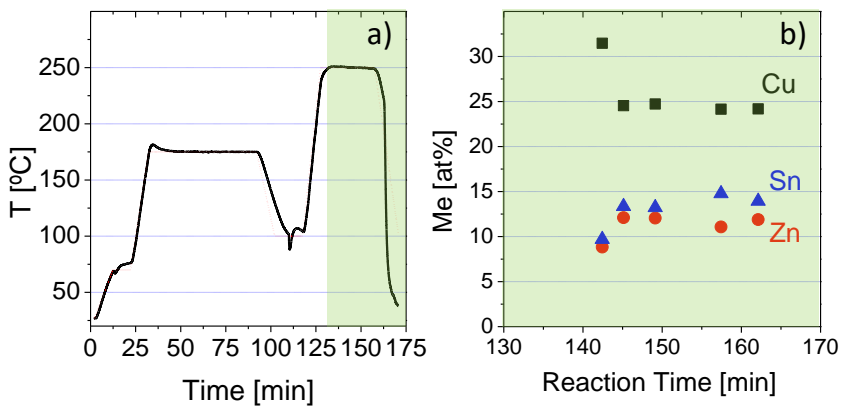

Figure 2. a) Programed (red) and real (black) temperature profiles; b) evolution of the composition of the CZTS nanocrystals during the synthesis: $\boldsymbol{C u}$; $\bullet \mathrm{Zn} ; \boldsymbol{\Delta} \mathrm{Sn}$.

Surprisingly, the heating rate had no significant role on the size and composition of the formed CZTS nanocrystals. For example, constant-rate 10 or 30 minutes ramps from $100{ }^{\circ} \mathrm{C}$ to $250{ }^{\circ} \mathrm{C}$ resulted in essentially the same final material. However, the stability of the heating rate had profound effect on the size distribution of the obtained CZTS nanocrystals, as consistently observed in over 20 syntheses shown in figure 3 . We associate this result to a better separation of the nucleation and growth stages and to a higher temperature homogeneity of the solution with a steady heating rate. Practically, this result points to the need for accurate control and knowledge of the 
temperature profile of the nanocrystal synthesis. The second main factor determining the size distribution was mixing of the reagents. Intense argon bubbling (few bubbles per second, ca. $30 \mathrm{ml} / \mathrm{min}$ ) was found to be a key to obtain high quality CZTS nanocrystals. Argon bubbling facilitates both mixing and heat transfer during the heating-up process, as previously indicated by Lynch et al. for the heating-up synthesis of iron oxide nanocrsytals. ${ }^{31}$

Besides the scalability of the heating based synthesis, highly important is also the ability to synthesize at high concentrations and with high reaction yields. Hence this factor had also received a great attention in this study. Figure $4 \mathrm{a}$ shows a ternary diagram with data points corresponding to the actual composition of CZTS nanocrystals produced at gradually higher precursor concentrations, obtained by reducing the amount of solvent (ODE) and keeping same quantities of reagents. Higher precursor concentrations resulted in a drop of the zinc content and slight increase in Sn content in the final CZTS nanocrystals. These changes were then eliminated by re-adjusting the ratios of reagents increasing $\mathrm{ZnO}$ and decreasing $\mathrm{SnCl}_{4} \cdot 5 \mathrm{H}_{2} \mathrm{O}$ amounts. As a side effect of additional $\mathrm{ZnO}$, large polydisperse $\mathrm{ZnS}$-rich nanocrystals formed, that can be easily removed by centrifugation. On the other hand, the batch-to-batch composition dispersion was relatively low as shown in figure $4 \mathrm{~b}$, where the composition of 16 CZTS nanocrystal batches obtained using the same metal concentration is shown.

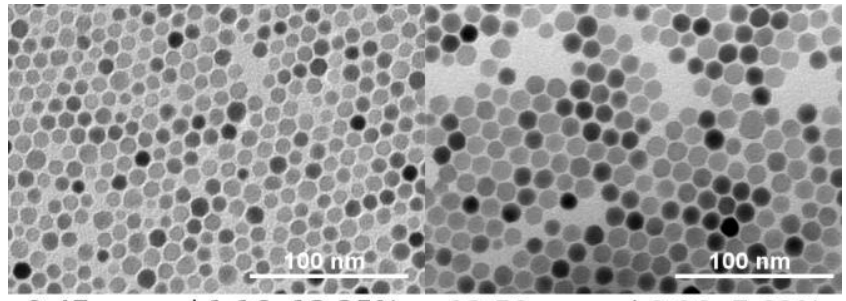

$9.47 \mathrm{~nm}$; sd $1.16 ; 12.25 \% \quad 12.58 \mathrm{~nm}$; sd $0.96 ; 7.62 \%$
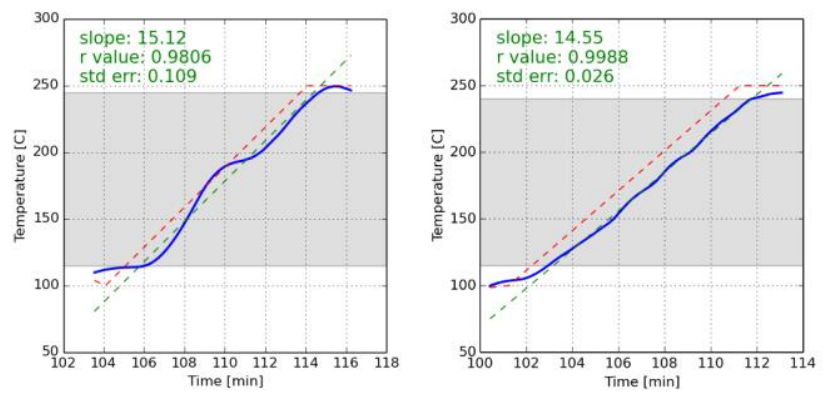

Figure 3. Representative TEM micrograph of the CZTS nanocrystals obtained from an irregular (left) and a steady (right) heating rate. CZTS nanocrystals with $12 \%$ size dispersion were obtained using a relatively irregular heating rate $(\mathrm{r}=0.9806)$. Nanocrystals produced with a more stable heating rate $(\mathrm{r}=0.9988)$ were characterized with size distributions of $7.6 \%$.
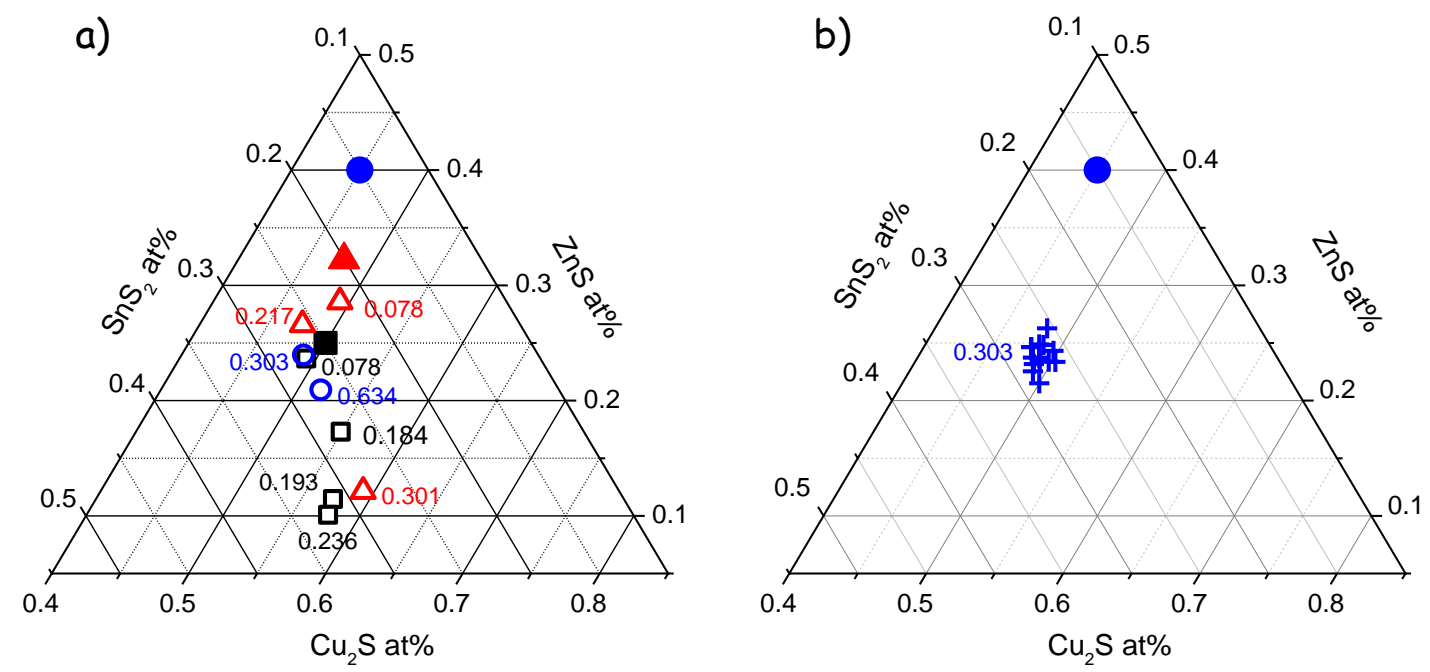

Figure 4. Ternary diagrams of the composition of CZTS nanocrystals. a) Composition for samples obtained using different total metal concentrations in the initial solution as detailed within the graph in mmol of the metals $\left(\Sigma_{(\mathrm{Cu}+\mathrm{Zn}+\mathrm{Sn})}\right)$ per gram of reaction mixture. The symbol color and shape display the initial ratio of metals. The [Cu, Zn, Sn] initial concentrations were: $\square=[2,1,1] ; \Delta=[2,1.35,0.84]$; and $\mathrm{O}=[2,1.78,0.67]$. The solid black square, red triangle and blue circle mark the initial precursor concentrations used for the materials represented with empty black squares, red triangles and blue circles, respectively. B) Composition of 16 CZTS samples produced with a metal concentration of $0.303 \mathrm{mmol} / \mathrm{g}$ and an initial metal ratio [Cu, $\mathrm{Zn}, \mathrm{Sn}]=[2,1.78,0.67]$.

The size, shape and monodispersity of CZTS nanocrystals did not significantly change with the total precursor concentration when maintaining the metal ratios constant. On the other hand, the $\mathrm{Cu}: \mathrm{Zn}: \mathrm{Sn}$ ratio was a main factor governing the shape of the CZTS nanoparticles. It is well known that the presence of tert-dodecylmercaptan in the reaction mixture is a key factor for the production of rodshaped CZTS and CTS nanoparticles. ${ }^{32}$ It is considered that while DDT binds equally to all facets, tDDM preferentially binds to specific facets, leaving the [001] direction for crystal growth. This difference allows the preparation of CZTS or
CTS nanocrystals with different aspect ratio. ${ }^{32}$ It is assumed that DDT binds to the surface of CZTS nanocrystals stronger, allowing the preparation of smaller but more homogeneous CZTS nanorods (See figure S3 for representative TEM micrographs of CZTS nanocrystals synthesized with and without DDT). Surprisingly, we found that at high precursor concentrations, the presence of tDDM did not significantly influence the shape of the CZTS nanoparticles. Only highly $\mathrm{Cu}$-rich precursor solutions in combination with high initial precursor concentration resulted in the formation of quasispherical CZTS nanocrystals, as shown in figure 5. 


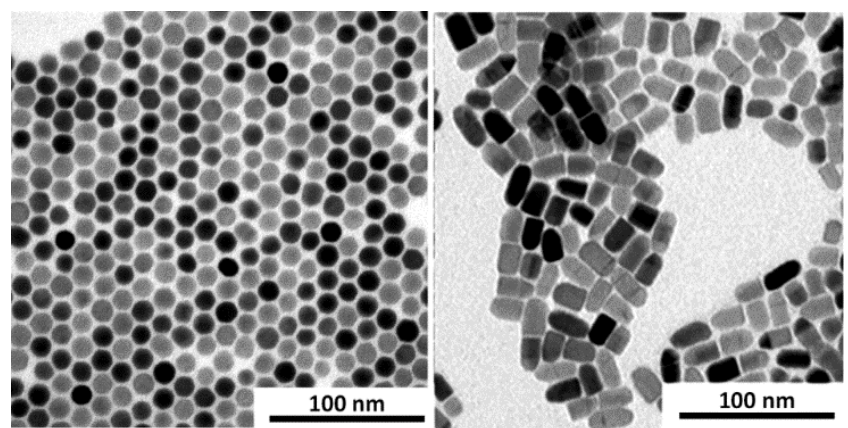

Figure 5. TEM micrograph of CZTS nanocrystals prepared using different $\mathrm{Cu}: \mathrm{Zn}: \mathrm{Sn}$ ratios and concentration; 3.50:2.67:1.00 and $0.303 \mathrm{mmol} / \mathrm{g}$ (left) and 2.40:1.60:1.00 and $0.219 \mathrm{mmol} / \mathrm{g}$ (right). TEM image of the rod-like CZTS nanocrystals prepared with stoichiometric precursor concentrations is presented on figure S2. The presence of tert-dodecylmercaptan in the reaction mixture did not influence the shape of CZTS nanocrystals at high precursor concentrations.

To study the surface chemistry of the synthesized CZTS nanocrystals, NMR measurements were performed on a purified dispersion of CZTS nanocrystals (Figure 6). In the ${ }^{1} \mathrm{H}$ NMR spectrum, overlapping sharp and broad resonances were detected in the chemical shift range 0-3 ppm (Figure 6a), rendering it difficult to directly extract information from that area. However, the isolated alkene resonance at $5.5 \mathrm{ppm}$ was clearly broadened, an effect which is attributed to nanocrystalligand binding. ${ }^{33}$ As OLA is the only molecule in the reaction mixture with an internal alkene, we thus identify OLA as ligand. Confirmation of the bound nature of OLA is provided by the negative (black) cross peaks between its resonances in the 2D NOESY spectrum (Figure 6b). In addition we find that the sharp resonances feature positive (red) cross peaks which means that the corresponding molecule does not interact with the surface.

The overlapping resonances at 0-3 ppm can be separated along the diffusion dimension (Figure 6c) as the broad resonances correspond to bound OLA, diffusing slowly while the sharp resonances belong a free, yet unidentified molecule, diffusing quickly. The diffusion coefficient of OLA is 41 $\mu \mathrm{m}^{2} / \mathrm{s}$ and can be converted to a solvodynamic diameter of 20 $\mathrm{nm}$ with the Stokes-Einstein equation. Since this value is in the same order of magnitude as the nanocrystal diameter, we conclude that OLA is tightly bound to the nanocrystal. ${ }^{34}$ Amines typically bind in a $\mathrm{NC}(\mathrm{L})$ binding motif (a stoichiometric nanocrystal with an L-type ligand coordinating to surface metal ions) and although they are generally considered as weakly binding ligands, there were found to bind tightly to copper indium sulfide nanocrystals. ${ }^{35}$ Here, we find the same tight binding to CZTS nanocrystals and we infer that this is due to the strong affinity of amines for copper.
Interestingly, neither the reference spectrum of DDT, nor the reference spectrum of tDDM match the sharp resonances of the unbound compound. However, when DDT is added to the sample, its resonances disappear after two weeks and the signals of the unidentified species have increased in intensity, suggesting that this compound is a degradation product of DDT.

Finally, the OLA concentration was quantified to $5.34 \mathrm{mM}$ and with the nanocrystal diameter $(13 \mathrm{~nm})$ and the nanocrystal concentration $(13 \mathrm{mM})$ we calculated a ligand density of 0.8 $\mathrm{nm}^{-2}$. This is a rather low value since full coverage typically corresponds to 4-5 ligands $\mathrm{nm}^{-2}$ and it could be explained by assuming that the original ligand shell comprised both thiol and amine and that the thiol degraded over time, possibly even catalyzed by the CZTS nanocrystals. However, to confirm these speculations, a thorough surface chemistry study during all the synthetic steps would be needed, which is outside the scope of this contribution. The solvodynamic diameter from the DOSY experiment $(20 \mathrm{~nm})$ was also higher than the sum of the nanocrystal core diameter $(13 \mathrm{~nm})$ and the organic ligand shell $(3 \mathrm{~nm})$. This leads us to believe that the nanocrystals agglomerate during the drying step - prior to dissolution in deuteroform - due to the low ligand coverage.

Potential applications of CZTS nanocrystals requires displacement of the bulky ligands from the surface of nanocrystals. A number of potentially useful short surfactants were tested as stabilizers of the CZTS nanoparticles in polar media (Table S1). Thermogravimetric analysis (SI) showed that weight loss during heat treatment was only a few \% and mainly depended on the drying condition (See supporting information for the details). Note that DMF and water are tightly bound to the surface of CZTS nanocrystals and an intensive drying procedure needs to be applied in order to remove them completely. CZTS nanocrystals typically loose solubility after such an excessive drying. Exchanged samples showed exceptionally low carbon content $(<1 \mathrm{w} \%$ with a record value of 0.1-0.2 w\% which is the practical limit of the used analytical instrument) after heat treatment in a nitrogen atmosphere. We believe that the residual carbon content depended more on the practical accuracy during cleaning procedure after ligand exchange than on the nature of the used stabilizer/solvent couple.

In summary, we detailed an over $90 \%$ yield procedure to prepare highly monodisperse wurtzite CZTS nanocrystals with size distributions below a $10 \%$. The synthesis protocol was easily scalable to the preparation of several grams of CZTS nanocrystals per batch. To produce stoichiometric nanocrystals with high concentration precursor solutions, initial metal ratios had to be adjusted. Finally, effective mixing using intense argon bubbling and a careful control of the heating ramp stability were found to be key parameters to produce highly monodisperse CZTS nanocrystals. 
a)

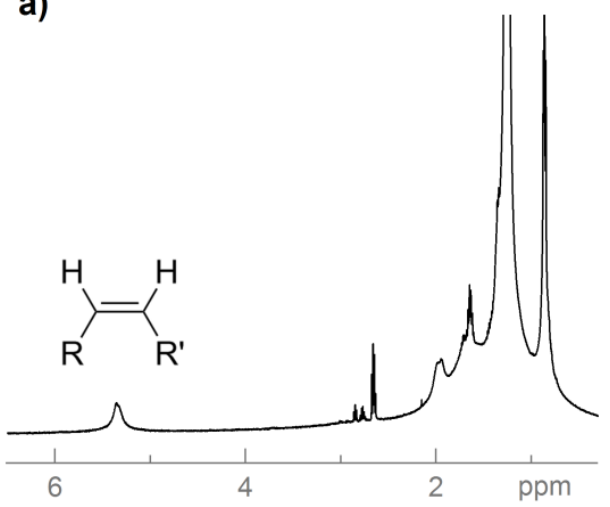

b)

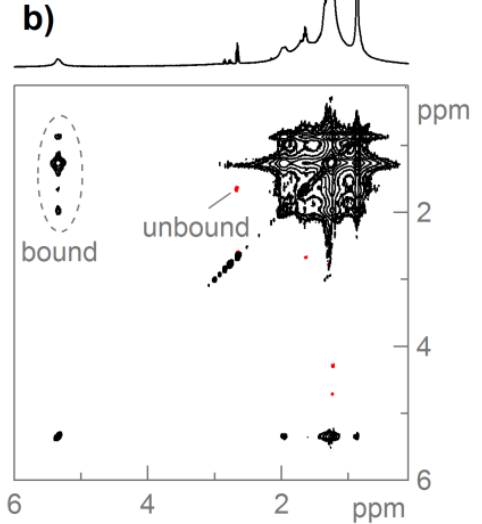

c)

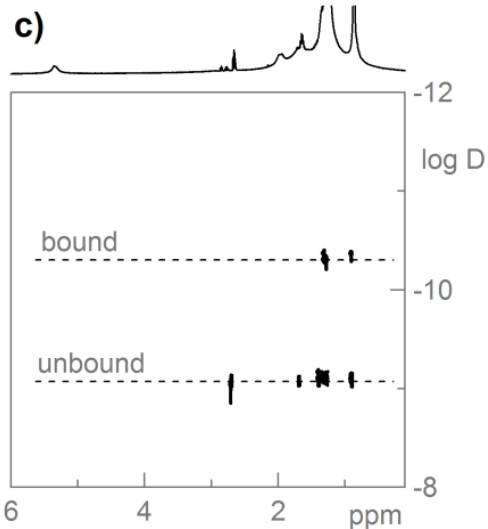

Figure 6: a) ${ }^{1} \mathrm{H}$ NMR spectrum of $14 \mathrm{~nm}$ CZTS nanocrystals in $\mathrm{CDCl}_{3}$, concentration is $8.4 \mu \mathrm{M}$ particles. b) NOESY spectrum of the same sample. c) DOSY spectrum of the same sample.

\section{ASSOCIATED CONTENT}

Supporting Information. Ligand exchange procedure, TEM images of CZTS nanoparticles prepared with and without tertdodecylmercaptan; TG, zeta potentials and IR spectra of the CZTS nanoparticles; additional HRTEM images of CZTS nanoparticles. This material is available free of charge via the Internet at http://pubs.asc.org.

\section{AUTHOR INFORMATION}

\section{Corresponding Author}

*ashavel@medcomadvance.com

\section{Present Addresses}

h Medcom Advance SA, Edifici N5, Campus de Sescelades, Carrer de Marcel·lí Domingo s/n, 43007 Tarragona, Spain.

\section{Author Contributions}

The manuscript was written through contributions of all authors. I All authors have given approval to the final version of the manuscript.

\section{ACKNOWLEDGMENT}

This work was supported by the European Regional Development Funds and the Framework 7 program under projects SCALENANO (FP7-NMP-ENERGY-2011-284486) and KESTCELLS (FP7/2007-2013/316488). MI thanks AGAUR for their Beatriu i Pinós postdoctoral grant (2013 BP-A00344). JDR thanks the FWO (Research Foundation Flanders) for financial support. M. V. K. acknowledges partial financial support by the European Union (EU) via FP7 ERC Starting Grant 2012 (Project NANOSOLID, GA No. 306733)Authors also acknowledge the funding from Generalitat de Catalunya 2014 SGR 1638.

\section{ABBREVIATIONS}

CZTS, $\left(\mathrm{Cu}_{2} \mathrm{ZnSnS}_{4}\right)$; ODE, 1-octadene; OLA, oleylamine; THF, tetrahydrofuran; t-DDT , tert-dodecyl mercaptan;

\section{REFERENCES}

1. Todorov, T. K.; Tang, J.; Bag, S.; Gunawan, O.; Gokmen, T.; Zhu, Y.; Mitzi, D. Beyond 11\% Efficiency: Characteristics of Stateof-the-Art $\mathrm{Cu}_{2} \mathrm{ZnSn}(\mathrm{S}, \mathrm{Se})_{4}$ Solar Cells. Adv. Energy. Mater. 2013, 3 , 34-38.

2. Chen, S.; Walsh, A.; Gong, X.-G.; Wei, S.-H. Classification of Lattice Defects in the Kesterite $\mathrm{Cu}_{2} \mathrm{ZnSnS}_{4}$ and $\mathrm{Cu}_{2} \mathrm{ZnSnSe} 4$ EarthAbundant Solar Cell Absorbers. Adv. Mater. 2013, 25, 1522-1539.

3. Carrete, A.; Shavel, A.; Fontané, X.; Montserrat, J.; Fan, J.; Ibáñez, M.; Saucedo, E.; Pérez-Rodrguez, A.; Cabot, A. Antimony-
Based Ligand Exchange To Promote Crystallization in SprayDeposited $\mathrm{Cu}_{2} \mathrm{ZnSnSe}_{4}$ Solar Cells. J. Am. Chem. Soc. 2013, 135, 15982-15985.

4. Wang, P.; Minegishi, T.; Ma, G.; Takanabe, K.; Satou, Y.; Maekawa, S.; Kobori, Y.; Kubota, J.; Domen, K. Photoelectrochemical Conversion of Toluene to Methylcyclohexane as an Organic Hydride by $\mathrm{Cu}_{2} \mathrm{ZnSnS}_{4}$-Based Photoelectrode Assemblies. J. Am. Chem. Soc. 2012, 134, 2469-2472.

5. Patra, B. K.; Shit, A.; Guria, A. K.; Sarkar, S.; Prusty, G.; Pradhan, N. Coincident Site Epitaxy at the Junction of $\mathrm{Au}-$ $\mathrm{Cu}_{2} \mathrm{ZnSnS}_{4}$ Heteronanostructures. Chem. Mater. 2015, 27, 650-657.

6 .Yu, X.; Shavel, A.; An, X.; Luo, Z.; Ibáñez, M.; Cabot, A. $\mathrm{Cu}_{2} \mathrm{ZnSnS}_{4}-\mathrm{Pt}$ and $\mathrm{Cu}_{2} \mathrm{ZnSnS}_{4}$-Au Heterostructured Nanoparticles for Photocatalytic Water Splitting and Pollutant Degradation. J. Am. Chem. Soc. 2014, 136, 9236-9239.

7. Yu, X; An, X.; Shavel, A.; Ibáñez M.; Cabot, A. The Effect of the Ga Content on the Photocatalytic Hydrogen Evolution of CuIn 1 . ${ }_{x} \mathrm{Ga}_{x} \mathrm{~S}_{2}$ nanocrystals. J. Mater. Chem. A 2014, 2, 12317-12322.

8. Yu, X.; Liu, J; Genç, A.; Ibáñez, M.; Luo, Z.; Shavel, A.; Arbiol, J.; Zhang, G.; Zhang, Y.; Cabot, A. $\mathrm{Cu}_{2} \mathrm{ZnSnS}_{4}-\mathrm{Ag}_{2} \mathrm{~S}$ Nanoscale $\mathrm{p}-\mathrm{n}$ Heterostructures as Sensitizers for Photoelectrochemical Water Splitting. Langmuir 2015, 31, 10555-10561.

9. Yu, X.; An, X.; Genç, A.; Ibáñez, M.; Arbiol, J.; Zhang, Y.; Cabot, A. $\mathrm{Cu}_{2} \mathrm{ZnSnS}_{4}-\mathrm{PtM}(\mathrm{M}=\mathrm{Co}, \mathrm{Ni})$ Nanoheterostructures for Photocatalytic Hydrogen Evolution. J. Phys. Chem. C 2015, 119, 21882-21888.

10. Liu, M.-L.; Chen, I.-W.; Huang, F.-Q.; Chen, L.-D. Improved Thermoelectric Properties of Cu-Doped Quaternary Chalcogenides of $\mathrm{Cu}_{2} \mathrm{CdSnSe}_{4}$. Adv. Mater. 2009, 21, 3808-3812.

11. Xiao, C.; Li, K.; Zhang, J.; Tong, W.; Liu, Y.; Li, Z.; Huang, P.; Pan, B.; Su, H.; Xie, Y. Magnetic Ions in Wide Band Gap Semiconductor Nanocrystals for Optimized Thermoelectric Properties. Mater. Horiz. 2014, 1, 81-86.

12. Guo, Q.; Hillhouse, H. W., Agrawal, R. Synthesis of $\mathrm{Cu}_{2} \mathrm{ZnSnS}_{4}$ Nanocrystal Ink and its Use for Solar Cells. J. Am. Chem. Soc. 2009, 131, 11672-11673.

13. Ibáñez, M.; Zamani, R.; Gorsse, S.; Fan, J.; Ortega, S.; Cadavid, D.; Morante, J. R.; Arbiol, J. Cabot, A. Core-Shell Nanoparticles As Building Blocks for the Bottom-Up Production of Functional Nanocomposites: $\mathrm{PbTe}-\mathrm{PbS}$ Thermoelectric Properties. ACS Nano 2013, 7, 2573-2586.

14. Ibáñez, M.; Korkosz, R. J.; Luo, Z.; Riba, P.; Cadavid, D.; Ortega, S.; Cabot, A. Kanatzidis, M. G. Electron Doping in BottomUp Engineered Thermoelectric Nanomaterials through HCl-Mediated Ligand Displacement. J. Am. Chem. Soc. 2015, 137, 4046-4049.

15. Ibáñez, M.; Zamani, R.; Lalonde, A.; Cadavid, D.; Li, W.; Shavel, A.; Arbiol, J.; Morante, J. R.; Gorsse, S.; Snyder, G. J. Cabot, A. $\mathrm{Cu}_{2} \mathrm{ZnGeSe}_{4}$ Nanocrystals: Synthesis and Thermoelectric Properties. J. Am. Chem. Soc. 2012, 134, 4060-4063.

16. Yang, W.-C.; Miskin, C. K.; Carter, N. J.; Agrawal, R., Stach, E. A. Compositional Inhomogeneity of Multinary Semiconductor 
Nanoparticles: A Case Study of $\mathrm{Cu}_{2} \mathrm{ZnSnS}_{4}$. Chem. Mater. 2014, 26 , 6955-6962.

17. Haas, W.; Rath, T.; Pein, A.; Rattenberger, J.; Trimmel, G., Hofer, F. The Stoichiometry of Single Nanoparticles of Copper Zinc Tin Selenide. Chem. Comm, 2011, 47, 2050-2052.

18. Shavel, A.; Cadavid, D.; Ibáñez, M.; Carrete, A., Cabot, A. Continuous Production of $\mathrm{Cu}_{2} \mathrm{ZnSnS}_{4}$ Nanocrystals in a Flow Reactor. J. Am. Chem. Soc. 2012, 134, 1438-1441.

19. Singh, A.; Geaney, H.; Laffir, F.; Ryan, K. M. Colloidal Synthesis of Wurtzite $\mathrm{Cu}_{2} \mathrm{ZnSnS}_{4}$ Nanorods and Their Perpendicular Assembly. J. Am. Chem. Soc. 2012, 134, 2910-2913.

20. van Embden, J.; Chesman, A. S. R.; Jasieniak, J. J. The HeatUp Synthesis of Colloidal Nanocrystals Chem. Mater. 2015, 27, 22462285.

21. Park, B.-I.; Hwang, Y.; Lee, S. Y.; Lee, J.-S.; Park, J.-K.; Jeong, J.; Kim, J. Y.; Kim, B.; Cho, S.-H.; Lee, D.-K. Solvent-Free Synthesis of $\mathrm{Cu}_{2} \mathrm{ZnSnS}_{4}$ Nanocrystals: a Facile, Green, Up-Scalable Route for Low Cost Photovoltaic Cells. Nanoscale 2014, 6, 11703 11711.

22. Chesman, A. S. R.; van Embden, J.; Duffy, N. W.; Webster, N. A. S.; Jasieniak, J. J. In Situ Formation of Reactive Sulfide Precursors in the One-Pot, Multigram Synthesis of $\mathrm{Cu}_{2} \mathrm{ZnSnS}_{4}$ Nanocrystals. Cryst. Growth Des. 2013, 13, 1712-1720.

23 10c) Chesman, A. S. R.; Duffy, N. W.; Peacock, S.; Waddington, L.; Webster, N. A. S. Jasieniak, J. J. Non-injection synthesis of $\mathrm{Cu}_{2} \mathrm{ZnSnS}_{4}$ nanocrystals using a binary precursor and ligand approach. $R S C A d v$., 2013, 3, 1017-1020.

24. Connell, M. A.; Bowyer, P. J.; Bone, P. A.; Davis, A. L.; Swanson, A. G.; Nilsson, M.; Morris, G. A. Improving the Accuracy of Pulsed Field Gradient NMR Diffusion Experiments: Correction for Gradient Non-Uniformity. J. Magn. Reson. 2009, 198, 121-131.

25. Sinnaeve, D. The Stejskal - Tanner Equation Generalized for Any Gradient Shape - an Overview of Most Pulse Sequences Measuring Free Diffusion. Concepts Magn. Reson. Part A 2012, 40, 39-65.

26. Ibáñez, M.; Zamani, R.; Li, W.; Shavel, A.; Arbiol, J.; Morante, J. R. Cabot, A. Extending the Nanocrystal Synthesis Control to Quaternary Composition. Cryst. Growth Des. 2012, 12, 1085-109.

27. Coughlan, C.; Ryan, K. M. Complete Study of the Composition and Shape Evolution in the Synthesis of $\mathrm{Cu}_{2} \mathrm{ZnSnS}_{4}$ (CZTS) Semiconductor Nanocrystals. CrystEng Comm, 2015, 17, 6914-6922.

28. Liao, H.-C.; Jao, M.-H.; Shyue, J.-J.; Chen, Y.-F.; Su, W.-F. Facile Synthesis of Wurtzite Copper-Zinc-Tin Sulfide Nanocrystals from Plasmonic Djurleite Nuclei. J. Mater. Chem. A 2013, 1, 337341.

39. Collord, A. D.; Hillhouse, H. W. Composition Control and Formation Pathway of CZTS and CZTGS Nanocrystal Inks for Kesterite Solar Cells. Chem. Mater. 2015, 27, 1855-1862.

30. Gabka, G.; Bujak, P.; Gryszel, M.; Ostrowski, A.; Malinowska, K.; Zukowska, G. Z.; Agnese, F.; Pron, A.; Reiss, P. Synthesis and Surface Chemistry of High Quality Wurtzite and Kesterite $\mathrm{Cu}_{2} \mathrm{ZnSnS}_{4}$ Nanocrystals Using Tin(ii) 2-Ethylhexanoate as a New Tin Source. Chem. Comm. 2015, 51, 12985-12988.

31. Lynch, J.; Zhuang, J.; Wang, T.; LaMontagne, D.; Wu, H.; Cao, Y. C. Gas-Bubble Effects on the Formation of Colloidal Iron Oxide Nanocrystals. J. Am. Chem. Soc. 2011, 133, 12664-12674.

32. Wang, J.-J.; Liu, P.; Ryan, K. M. A Facile Phosphine-Free Colloidal Synthesis of $\mathrm{Cu}_{2} \mathrm{SnS}_{3}$ and $\mathrm{Cu}_{2} \mathrm{ZnSnS}_{4}$ Nanorods with a Controllable Aspect Ratio. Chem. Comm. 2015, 51, 13810-13813.

33. Hens, Z., Martins, J. C. A Solution NMR Toolbox for Characterizing the Surface Chemistry of Colloidal Nanocrystals. Chem. Mater. 2013, 25, 1211-1221.

34. De Roo, J.; Van den Broeck, F.; De Keukeleere, K.; Martins, J. C.; Van Driessche, I.; Hens, Z. Unravelling the Surface Chemistry of Metal Oxide Nanocrystals, the Role of Acids and Bases. J. Am. Chem. Soc. 2014, 136, 9650-9657.

35. Dierick, R.; Van den Broeck, F.; De Nolf, K.; Zhao, Q.; Vantomme, A.; Martins, J. C.; Hens, Z. Surface Chemistry of CuInS 2 Colloidal Nanocrystals, Tight Binding of L-Type Ligands. Chem. Mater. 2014, 26, 5950-5957.

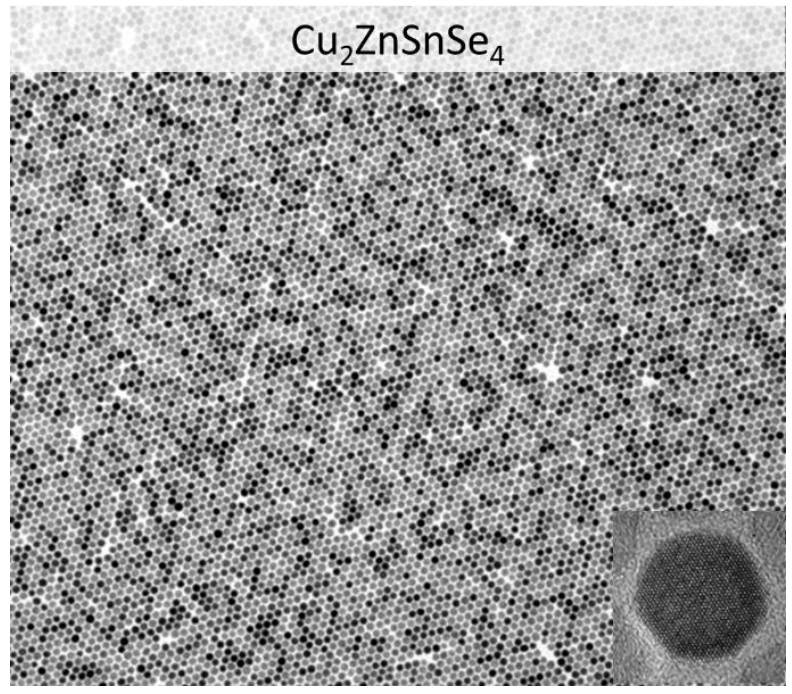

